\title{
Dynamic MRI Reconstruction as a Moment Problem
}

\author{
M. Zwaan \\ Centre for Mathematics and Computer Science, P.O. Box 4079, 1009 AB Amsterdam, \\ The Netherlands
}

Communicated by G. F. Roach

\begin{abstract}
This paper deals with some mathematical aspects of magnetic resonance imaging (MRI). MRI is used in diagnostic medicine to measure and display the cross section of, for example, a human organ. In this paper we formulate the MRI-reconstruction problem and solve it after restating it as a moment problem in a Hilbert space.
\end{abstract}

\section{Introduction}

In this paper we formulate and solve a reconstruction problem concerning magnetic resonance imaging (MRI). MRI is a diagnostic method for measuring and displaying the cross sections of human organs. We are particularly interested in the mathematical aspects of MRI reconstruction in the case of the beating human heart.

In section 1 we explain a strategy, called retrospective synchronization, to measure MRI data in the case of the beating human heart. At the end of this first section we state the reconstruction problem for dynamic MRI.

Section 2 is devoted to a moment problem in a Hilbert space, and Riesz bases are used to obtain a solution. Section 3 solves the reconstruction problem after reformulating it as a moment problem in an $L^{2}$-space of vector-valued functions.

\section{Magnetic resonance imaging}

This section explains magnetic resonance imaging (MRI), which is a technique for measuring and displaying the proton density of a cross section of a human organ. At the end of this section a mathematical formulation of the problem is stated.

A well written book on the applications of MRI in biomedicine is the monograph by Mansfield and Morris [12]. For those who are interested in the physics of MRI, the paper [10] by Hinshaw and Lent is appropriate. Acquisition methods in the case of a beating human heart are given by Bohning in [4]. 


\subsection{Acquisition of data}

In this subsection we describe how the MRI data are obtained.

If we represent (in the time-independent case) the absolute value of the proton density of a cross section by means of a function $f: \mathbb{R}^{2} \rightarrow \mathbb{R}$ the MRI device (approximately) measures the Fourier coefficient of $f$

$$
\int_{D} f(x, y) \mathrm{e}^{-\mathrm{i}(\kappa x+\lambda y)} d \mu(x, y) .
$$

Here $\mu$ is the Lebesgue measure, and $D \subset \mathbb{R}^{2}$ is the support of the function $f$.

In practice it is only possible to find the Fourier coefficient $\hat{f}$ of a function $f$ at a finite number of frequencies; to be somewhat more specific, $\kappa=0, \ldots, 255$; $\lambda=0, \ldots, 255$. Assume that this sequence can be measured instantaneously (in practice this may take from 2 up to $10 \mathrm{~ms}$ ). One such sequence of measurements $\{\hat{f}(\kappa, \lambda)\}_{\kappa=0, \ldots, 255}$, for fixed $\lambda$ is called a profile.

If we want to use MRI to measure and display cross sections of the beating human heart, then we have to consider a function that not only depends on the variable $(x, y)$ but also on the time $T$. So, in the following we want to consider a function $F(x, y, T)$, which we can think of as the absolute value of the proton density of a cross section of a beating heart. The reason why the function and the time are denoted by capitals will become clear later.

Before describing an acquisition method that is used in practice for measuring the Fourier transform of the proton density of a beating heart, we first give some terminology.

(i) An R-pulse is the electric pulse in the heart that marks the beginning of a heartbeat. It is recorded by means of an ECG, simultaneously with the measurements.

(ii) $A R R$-interval is the duration (in seconds) between two consecutive $\mathrm{R}$ pulses.

(iii) $A$ unit $R R$-interval is an $R R$ interval of one unit time length, say one second, which will be used as a reference interval, this interval is called $J$.

(iv) An heart phase is a phase in the periodic movement of the heart.

We explain an acquisition method, which we shall call retrospective synchronization, as described by Bohning [4] under the name retrospective gating. First introduce a function $f: \mathbb{R}^{2} \times J \rightarrow \mathbb{R}$, which we define as the standard heartbeat. Here $J$ is the unit $R R$ interval. We assume that the heart, during each heartbeat, is a rescaled copy of the function $f$ in time; this rescaling should be based on a biological model of the movement of the heart. In order to give an example, we assume the rescaling to be linear. Suppose the $k$ th $R$-pulse is measured at the time $r_{k}$, for $k=1,2, \ldots$ Assume that the proton density in a cross section of the beating heart, $F(x, y, T)$, is given in terms of $f$ as

$$
F(x, y, T):=f\left(x, y, \frac{T-r_{k}}{r_{k+1}-r_{k}}\right),
$$

where $T \in\left[r_{k}, r_{k+1}\right)$. This so-called dynamic case is more complex than the static case; in the dynamic case we measure the Fourier coefficient of a function $F$ at a certain time $T_{i}, \hat{F}\left(\kappa, \lambda, T_{i}\right)$. The profile that is measured for fixed $\lambda$, at time $T_{i}$, is denoted as 
$\left\{\hat{F}\left(\kappa, \lambda, T_{i}\right)\right\}_{\kappa=0 \ldots 255}$. In order to reconstruct the function $F$ at time $T_{i}$ by means of the Fourier inversion formula, we should measure the 256 profiles (i.e. for $\lambda=0, \ldots, 255$ ) at the time $T_{i}$. In practice one cannot measure quickly enough with MRI to obtain all these profiles during one heart phase, but they are obtained during several $R R$ intervals. The aim of MRI in the dynamic case is not to give a real-time reconstruction of the beating heart during different $R R$ intervals, but to reconstruct the standard heartbeat. That is, we have to translate our measurements in terms of the function $f$. In the case of linear rescaling this can be done as in formula (1.2), if $T_{i} \in\left[r_{k}, r_{k+1}\right)$ then we define

$$
t_{i}:=\frac{T_{i}-r_{k}}{r_{k+1}-r_{k}}
$$

and $\hat{f}\left(\kappa, \lambda, t_{i}\right):=\hat{F}\left(\kappa, \lambda, T_{i}\right)$. The variable $t_{i}$ lies in the unit heart interval. The rescaling from $T_{i}$ to $t_{i}$ is called projection onto the unit heart interval. After this the $t_{i}$ are reordered to obtain an increasing sequence.

The profiles are obtained as follows. We fix $\lambda$ and we measure the profile $\left\{\hat{F}\left(\kappa, \lambda, T_{1}\right)\right\}_{\kappa=0 \ldots 255}$, briefly denoted as $\left\{\hat{F}\left(\kappa, \lambda, T_{1}\right)\right\}$, at time $T_{1}$. After some time (this may be from 10 up to $200 \mathrm{~ms}$ ) we again measure a profile for $\lambda$, at time $T_{2}$, etc; the time at which the measurements take place is recorded. After we have obtained a fixed number of profiles (in practice this may be up to 80 ) the value of $\lambda$ is increased. If an $\mathrm{R}$ pulse has occurred, it is registered, so that, afterwards, the measured profile can be assigned to the corresponding heart phase. This is done as follows.

(1) The length of the heartbeat (i.e. the RR interval), in which the measurement under consideration occurred is computed. The time of a measurement, relative to the unit RR interval is computed, for example, in the case of linear rescaling by (1.3), and the data are projected onto this interval. (cf. Fig. 1.) We remark that the $t_{i}$ depend on the value of $\lambda$, in the sense that other values for $\lambda$ will give rise to another arrangement of the $t_{i}$ on the unit $\mathrm{RR}$ interval. To express this dependence we shall denote the time as $t_{i}(\lambda)$.



Fig. 1. Diagram showing how the measurements are projected onto a unit RR-interval 
(2) If we want to display the heart at several phases, the unit RR interval is subdivided into several parts (see Fig. 1, for the case when the RR interval is subdivided into four parts). All profiles on the unit RR interval between phase 1 and phase 2 are, in practice, considered to be measured at phase 1. All measurements between phases 2 and 3 are considered to be measured at phase 2 , etc. If several measurements belong to phase $n$, then the average of these is assigned to phase $n$, that is in practice one uses interpolation by zeroth-order splines to obain data at the phases. We want to improve this reconstruction algorithm by using another type of interpolation. In order to do this, definition of the problem is given in mathematical terms, in the following subsection.

\subsection{Definition of a mathematical problem}

Before giving a definition of the problem, we first introduce some notation. We change our conventions slightly by writing $x$ and $\kappa$ instead of the pairs $(x, y)$ and $(\kappa, \lambda)$. Let $\mathbb{K}$ and $\mathbb{Q}$ be finite or countable index sets, for example, $\mathbb{K}:=\{0, \ldots, 255\}^{2}$ or $\mathbb{K}:=\mathbb{Z}^{2}$, and $\mathbb{\mathbb { V }}:=\{1, \ldots, I\}$, or $\mathbb{\mathbb { V }}:=\mathbb{Z}$. Let $D \subset \mathbb{R}^{2}$ be the unit square $D=[-\pi, \pi]^{2}$. Suppose the object to be measured has support in this interval $D$. The function

$$
f: D \times \mathbb{R} \ni(x, t) \rightarrow f(x, t)
$$

represents a two-dimensional cross section of the beating heart, during a standard heartbeat. The Fourier coefficient of $f$, taken with respect to the variable $x$ is defined by

$$
\hat{f}(\kappa, t):=\frac{1}{2 \pi} \int_{D} f(x, t) \mathrm{e}^{-\mathrm{i} \kappa x} d \mu(x) .
$$

The data $\left\{\hat{f}\left(\kappa, t_{i}(\kappa)\right)\right\}_{\kappa \in K}$ are measured, for fixed $\kappa$, at the rescaled time $t_{i}(\kappa)$, for $i \in \mathbb{\Xi}$. Suppose we have measured

$$
g_{\kappa, i}:=\hat{f}\left(\kappa, t_{i}(\kappa)\right)
$$

for $\kappa \in \mathbb{K}$ and $i \in \mathbb{Q}$. The problem is to find a function $f: D \times \mathbb{R} \rightarrow \mathbb{R}$ such that

$$
\hat{f}\left(\kappa, t_{i}(\kappa)\right)=g_{\kappa, i},
$$

for $\kappa \in \mathbb{K}$, and $i \in \mathbb{Q}$. We refer to problem (1.5) as a mixed Fourier-interpolation problem.

In the following section we solve this mixed problem for both finite and countable index sets $\mathbb{K}$ and $\mathbb{U}$.

\section{The moment problem in Hilbert space}

This section considers a moment problem in a Hilbert space, and uses Riesz bases to obtain a solution.

The theory of moment problems is dealt with in the following books. Akhiezer [1] gives an overview of several types of moment problems. The theory of the generalized 
inverse to the moment problem in a Hilbert space is applied by Bertero [3]. Young [16] uses the theory of Riesz bases and Riesz-Fischer systems applied to the moment problem in a Hilbert space. The moment problem in reproducing kernel Hilbert spaces is considered by Shapiro [15] and Landau [11] gives an overview of the applications and theory of moment problems.

The notational convention used throughout this paper is as follows. The Fourier transform of a function $f$ is denoted by $\hat{f}$. The frequency parameters are written as Greek symbols, like $\xi, \kappa$, the time variable is $t$ and $z$ denotes complex number. The inner product of a Hilbert space $\mathscr{H}$ is denoted by $\langle,\rangle_{\mathscr{H}}$. The index sets $\mathbb{K}$ and $\mathbb{Q}$ are equal to $\mathbb{Z}^{2}$ and $\mathbb{Z}$, respectively, unless stated otherwise. $\left\{h_{i}\right\}_{i \in \mathbb{l}}$ and $\left\{e_{\kappa}\right\}_{\kappa \in \kappa}$ are orthonormal bases for $\mathscr{H}$ and $L^{2}(D)$, respectively.

\subsection{Interpolation as a moment problem}

In this subsection we consider interpolation and moment problems. It turns out that an interpolation problem is a special type of moment problem. that

Define the space $l^{2}(\mathbb{0})$ as the set of all sequences of complex numbers $\left\{g_{i}\right\}_{i \in \mathbb{1}}$ such

$$
\|g\|_{l^{2}}^{2}:=\sum_{i \in \emptyset}\left|g_{i}\right|^{2}<\infty
$$

Suppose we have measured a sequence of complex numbers $\left\{g_{i}\right\} \in l^{2}(\mathbb{0})$ at the points in time $t_{i}$. The interpolation problem is to find a function $f: \mathbb{R} \rightarrow \mathbb{R}$ that satisfies

$$
\sqrt{(\pi / r)} f\left(t_{i} \pi / r\right)=g_{i}, \quad \forall i \in \mathbb{Q}
$$

(The reason for the factor $\pi / r$ will become clear at the end of this section.) Furthermore we want the function $f$ to lie in the Hilbert space of band-limited functions, denoted by $\mathbb{P}_{r}$. Denote the support of a function by supp.

Definition 2.1. $\mathbb{P}_{r}:=\left\{f \in L^{2}(\mathbb{R}) \mid \operatorname{supp} \hat{f} \subset[-r, r]\right\}$.

$\mathbb{P}_{r}$ becomes a Hilbert space with the inner product

$$
\langle f, g\rangle \mathbb{P}_{r}:=\int_{\mathbb{R}} f(t) \overline{g(t)} \mathrm{d} t .
$$

By the theorem of Paley-Wiener any $f \in \mathbb{P}_{r}$ can be extended to an analytic function such that

$$
|f(z)| \leqslant \mathrm{e}^{r|\operatorname{Im} z|}\|f\|_{\mathbb{P}}, \quad \forall z \in \mathbb{C} .
$$

By the above inequality, it follows that convergence in $\mathbb{P}_{r}$ implies uniform convergence on horizontal strips in $\mathbb{C}$.

$\left\{\sqrt{ }(r / \pi) \operatorname{sinc}_{r}(t-n \pi / r)\right\}_{n \in \mathbb{1}}$ is an orthonormal basis for $\mathbb{P}_{r}$, where the sinc function is given by

$$
\operatorname{sinc}_{r}(t):=\left\{\begin{array}{cc}
\sin (r t) / r t, & t \neq 0 \\
1, & t=0
\end{array}\right.
$$


The point evaluation at $t_{i}$ can be written in terms of a sinc function. Let

$$
\varphi_{i}:=\sqrt{ }(r / \pi) \operatorname{sinc}_{r}\left(\cdot-t_{i} \pi / r\right)
$$

then for all $f \in \mathbb{P}_{r}$,

$$
\left\langle f(\cdot), \varphi_{i}\right\rangle_{\mathbb{P}_{r}}=\sqrt{ }(\pi / r) f\left(t_{i} \pi / r\right) .
$$

With this equality the interpolation problem is a special kind of moment problem in a Hilbert space $\mathscr{H}$, which can be formulated as follows. Let $\left\{\varphi_{i}\right\}_{i \in l}$ be a sequence of vectors in $\mathscr{H}$. Suppose $\left\{g_{i}\right\} \in l^{2}(\mathbb{0})$. We want to find an element $f$ of $\mathscr{H}$ such that

$$
\left\langle f, \varphi_{i}\right\rangle_{\mathscr{H}}=g_{i}, \quad \forall i \in \mathbb{U} \text {. }
$$

\subsection{Riesz bases}

In this section we find a solution to the moment problem in a Hilbert space $\mathscr{H}$, which is a solution to the interpolation problem (2.1) in the case that $\mathscr{H}=\mathbb{P}_{r}$.

Without conditions on the system $\left\{\varphi_{i}\right\}$ the moment problem need not have a solution. A sufficient condition for (2.4) to have a solution is that $\left\{\varphi_{i}\right\}$ is a Riesz basis (cf. Gohberg and Krein [8], or Young [16]).

Definition 2.2. A system of vectors $\left\{\varphi_{i}\right\}_{i \in 1} \subset \mathscr{H}$ is a Riesz basis if there is a bounded linear invertible operator $T$ on $\mathscr{H}$ such that

$$
T \varphi_{i}=h_{i}, \quad \forall i \in \mathbb{l} .
$$

A linear operator $T$ is called invertible if its inverse, denoted by $T^{-1}$, exists and is bounded. A Riesz basis $\left\{\varphi_{i}\right\}_{i \in \mathbb{l}}$ possesses a unique biorthogonal system, $\left\{\psi_{i}\right\}_{i \in \mathbb{l}}$, that is

$$
\left\langle\varphi_{i}, \psi_{j}\right\rangle_{\mathscr{H}}=\delta_{i j}, \quad \forall i, j \in \mathbb{\mathbb { I }} .
$$

It can be checked that

$$
\psi_{i}=T^{*} h_{i}, \quad \forall i \in \mathbb{Q} .
$$

Here $T$ and $h_{i}$ are as in Definition 2.2 and $T^{*}$ is the adjoint of $T .\left\{\psi_{i}\right\}$ is a Riesz basis, which can also be computed in terms of the bounded and invertible Gram matrix,

as

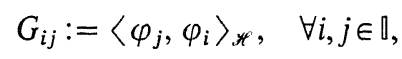

$$
\psi_{i}=\sum_{j \in \mathbb{1}}{\overline{\left(G^{-1}\right.}}_{i j} \varphi_{j}, \quad \forall i \in \mathbb{Q} .
$$

Note that this formula holds both in the case of finite and countable index sets. If a system of vectors $\left\{\varphi_{i}\right\} \subset \mathscr{H}$ is linearly independent and finite, then it is a Riesz basis for its linear span, $\mathscr{H}_{1}:=\operatorname{span}\left\{\varphi_{i}\right\}_{i \in l}$.

Throughout the remainder of this paper the system $\left\{\varphi_{i}\right\}_{i \in 1}$ is a Riesz basis for $\mathscr{H}$ and its associated biorthogonal system is denoted by $\left\{\psi_{i}\right\}_{i \in 1}$.

Any $f \in \mathscr{H}$ can be uniquely written as

$$
f=\sum_{i \in 1}\left\langle f, \varphi_{i}\right\rangle_{\mathscr{H}} \psi_{i} .
$$


Hence the moment problem (2.4) has the unique solution

$$
f=\sum_{i \in \mathbb{l}} g_{i} \psi_{i}
$$

if $\left\{g_{i}\right\} \in l^{2}(0)$.

In the case that $\left\{\varphi_{i}\right\}$ is a finite linearly independent system of vectors in $\mathscr{H}$, which is not a basis for $\mathscr{H}$, then $f$ (formula (2.6)) is a solution to the moment problem. It is not unique, since other solutions can be obtained by adding elements to $f$, which are orthogonal to $\operatorname{span}\left\{\varphi_{i}\right\}_{i \in \mathbb{I}}$. However, then the solution with the minimum norm among all the solutions is unique. In the following we give two examples of the solutions to the interpolation problem (2.1). The first deals with the case of a finite index set $\nabla$ and the second with an infinite (i.e. countable) index set. The theory of Riesz bases is not needed in the finite-dimensional case, but the formulae are in this case the same, and we include an example for illustration.

Example 2.1. Let $\mathbb{b}$ be a finite index set, say $\square=\{1, \ldots, I\}$ and let $t_{i}$ be a sequence of distinct real numbers, with $i \in \mathbb{Q}$. Let $r=\pi$. Consider the moment problem (2.1). We want to find a solution $f \in \mathbb{P}_{\pi}$ to (2.1) of minimum norm. If $f \in \mathbb{P}_{\pi}$ then we have

$$
\left\langle f(\cdot), \operatorname{sinc}_{\pi}\left(\cdot-t_{i}\right)\right\rangle_{\mathbb{P}_{\pi}}=f\left(t_{i}\right) .
$$

If the $t_{i}$ are distinct, then $\left\{\varphi_{n}\right\}_{n=1 \ldots 0}$ is linearly independent, so it is a Riesz basis for its linear span $\mathscr{H}_{1} \subset \mathbb{P}_{\pi}$. The minimum-norm solution to (2.1) in $\mathbb{P}_{\pi}$ is given by (2.6). $\psi_{n}$ can be computed with (2.5). Here $G$ is defined by

$$
G_{i j}:=\left\langle\varphi_{j}, \varphi_{i}\right\rangle_{\mathbb{P}_{\pi}}=\operatorname{sinc}_{\pi}\left(t_{i}-t_{j}\right), \quad \forall i, j \in \mathbb{Q} .
$$

The following example is concerned with an infinite sequence of vectors in $\mathbb{P}_{\pi}$.

Example 2.2. Let $\mathbb{\square}=\mathbb{Z}$. By Young [16], p. 42, Theorem 14 we have that $\left\{\varphi_{i}\right\}_{i \in l}$, given by

$$
\varphi_{i}:=\operatorname{sinc}_{\pi}\left(\cdot-t_{i}\right),
$$

is a Riesz basis for $\mathbb{P}_{\pi}$ if $t_{i}$ is a sequence of real numbers such that

$$
\left|t_{i}-i\right| \leqslant \alpha<1 / 4,
$$

for all $i \in \mathbb{l}$. The unique solution $f \in \mathbb{P}_{\pi}$ to the interpolation problem (2.1) is

$$
f=\sum_{i \in 0} g_{i} \psi_{i}
$$

where

$$
\psi_{i}=\sum_{j \in \mathbb{l}}{\overline{\left(G^{-1}\right.}}_{i j} \varphi_{j} .
$$

The Gram matrix $G$ is in this case,

$$
G_{i j}=\left\langle\varphi_{j}, \varphi_{i}\right\rangle_{p}=\operatorname{sinc}_{\pi}\left(t_{i}-t_{j}\right), \quad \forall i, j \in \mathbb{U} .
$$

If the $t_{i}$ satisfy (2.7), then it follows by the proof of Young [16], p. 38, Theorem 10 that there exists a bounded linear invertible operator $T$ on $\mathbb{P}_{r}$ such that

$$
T \varphi_{i}=h_{i}, \quad \forall i \in \mathbb{Q},
$$


and

$$
\|T\| \leqslant \frac{1}{1-\lambda}, \quad\left\|T^{-1}\right\| \leqslant 1+\lambda,
$$

where $\lambda:=1-\cos \pi \alpha+\sin \pi \alpha$,

$$
\varphi_{i}:=\sqrt{ }(r / \pi) \operatorname{sinc}_{r}\left(\cdot-t_{i} \pi / r\right), \quad \text { and } h_{i}:=\sqrt{ }(r / \pi) \operatorname{sinc}_{r}(\cdot-\mathrm{i} \pi / r) .
$$

\section{The moment problem in an $L^{2}$-space of vector-valued functions}

This section gives the mathematical theory to solve (1.5) by restating it as a moment problem in an $L^{2}$-space of vector-valued functions. This space, denoted by $L^{2}(D, \mathscr{H})$ consists of functions that map elements from $D$ into a Hilbert space $\mathscr{H}$. We extend the theory of Riesz bases to $L^{2}(D, \mathscr{H})$ and solve the mixed Fourier-interpolation problem (1.5).

Subsection 3.1 introduces the space $L^{2}(D, \mathscr{H})$. Riesz bases in this space are considered in subsection 3.2. In subsection 3.3 we use Riesz bases to solve a moment problem in $L^{2}(D, \mathscr{H})$ and subsection 3.4 applies the results in the case that $\mathscr{H}=\mathbb{P}_{r}$.

\subsection{The space $L^{2}(D, \mathscr{H})$}

In this subsection we consider $L^{2}$-spaces of functions that have values in a separable Hilbert space $\mathscr{H}$. For a more detailed discussion about these $L^{2}$-spaces, we refer to Balakrishnan [2] and Hille and Phillips [9].

A function that maps $D$ into $\mathscr{H}$ is called a vector-valued function. The notion of the measurability of such a function can be defined in terms of the Lebesgue measurability of complex-valued functions.

Definition 3.1. A function $f: D \rightarrow \mathscr{H}$, is called measurable, if for each $h \in \mathscr{H}$ the function $x \rightarrow\langle f(x), h\rangle_{\mathscr{H}}$ is Lebesgue measurable.

By Balakrishnan [2], the integral

$$
\int_{D}\|f(x)\|_{\mathscr{H}}^{2} \mathrm{~d} \mu(x)
$$

is well-defined in $\mathbb{R} \cup\{\infty\}$.

Identifying the functions that are equal up to a set of measure zero, we define the space $L^{2}(D, \mathscr{H})$ as the collection of measurable vector-valued functions such that (3.1) is finite. We write $\mathscr{W}=L^{2}(D, \mathscr{H})$ for short, and introduce the inner product $\langle\text {, }\rangle_{\text {w }}$ on $\mathscr{W}$, by

$$
\langle f, g\rangle_{\mathscr{W}}:=\int_{D}\langle f(x), g(x))_{\mathscr{H}} \mathrm{d} \mu(x) .
$$

It is proven in Balakrishnan [2], p. 134 that $\mathscr{W}$ with this inner product is a Hilbert space, with orthonormal basis $\left\{e_{\kappa} h_{i}\right\}_{\kappa \in K, i \in U}$, if $\left\{e_{\kappa}\right\}$ and $\left\{h_{i}\right\}$ are orthonormal bases for $L^{2}(D)$ and $\mathscr{H}$, respectively. By ' $e_{\kappa} h_{i}$ ' we denote the function that maps $x \in D$ onto the element $e_{\kappa}(x) h_{i} \in \mathscr{H}$. 
For $f \in L^{2}(D, \mathscr{H})$ an $\mathscr{H}$-valued integral of $f$, the Pettis integral, denoted by

$$
(\mathscr{P}) \int_{D} f(x) \mathrm{d} \mu(x)
$$

can be defined by its action on $h \in \mathscr{H}$,

$$
\left\langle(\mathscr{P}) \int_{D} f(x) \mathrm{d} \mu(x), h\right\rangle_{\mathscr{H}}:=\int_{D}\langle f(x), h\rangle_{\mathscr{H}} \mathrm{d} \mu(x) .
$$

Denote the (generalized) Fourier coefficient of a vector-valued function $f$, by

$$
\hat{f}(\kappa):=(\mathscr{P}) \int_{D} f(x) \overline{e_{\kappa}(x)} \mathrm{d} \mu(x) .
$$

Note that $\hat{f}: \mathbb{K} \rightarrow \mathscr{H}$. For the (generalized) Fourier transform Parseval's relation and an inversion formula are obtained by Foias and Nagy [6].

Proposition 3.2. Let $\mathscr{H}$ be a separable Hilbert space. For any $f \in L^{2}(D, \mathscr{H})$ the following identities hold:

$$
\begin{gathered}
\|f\|_{\mathscr{W}}^{2}=\sum_{\kappa \in \mathbb{K}}\|\hat{f}(\kappa)\|_{\mathscr{H}}^{2}, \\
f=\sum_{\kappa \in \mathbb{K}} \hat{f}(\kappa) e_{\kappa} .
\end{gathered}
$$

Here (3.3) converges in the norm topology.

\subsection{Riesz bases in $L^{2}(D, \mathscr{H})$}

In the previous section it turned out that $\left\{e_{\kappa} h_{i}\right\}$ is an orthonormal basis for $L^{2}(D, \mathscr{H})$. For a Riesz basis a similar statement holds, under some additional conditions.

If $\left\{\varphi_{\kappa, i}\right\}_{i \in \emptyset}$ is a Riesz basis in $\mathscr{H}$ for all $\kappa \in \mathbb{K}$, then by definition, there exists for each $\kappa \in \mathbb{K}$ a bounded linear invertible operator $T_{\kappa}$ on $\mathscr{H}$, with

$$
T_{\kappa} \varphi_{\kappa, i}=h_{i}, \quad \forall^{\times} i \in \| \text {. }
$$

A family $\left\{T_{\kappa}\right\}_{\kappa \in l}$ of operators is uniformly bounded if $\sup _{\kappa}\left\|T_{\kappa}\right\|<\infty$.

Proposition 3.3. Let $\left\{\varphi_{\kappa, i}\right\}_{i \in \mathbb{l}}$ be a Riesz basis in $\mathscr{H}$, for all $\kappa \in \mathbb{K}$. If $\left\{T_{\kappa}\right\}_{\kappa \in \mathbb{K}}$ and $\left\{T_{\kappa}^{-1}\right\}_{\kappa \in \mathbb{K}}$ are uniformly bounded families, then there is a bounded linear invertible operator $\mathscr{U}$ on $\mathscr{W}$ such that

$$
\mathscr{U}\left(e_{\kappa} \varphi_{\kappa, i}\right)=e_{\kappa} h_{i}, \quad \forall \kappa \in \mathbb{K}, \forall i \in \mathbb{U} .
$$

So $\left\{e_{\kappa} \varphi_{\kappa, i}\right\}$ is a Riesz basis for $\mathscr{W}$.

Proof. Suppose that for $\kappa \in \mathbb{K}$ fixed, $\left\{\varphi_{\kappa, i}\right\}$ is a Riesz basis for $\mathscr{H}$, such that we can find a family of uniformly bounded linear invertible operators, for which it holds that for $\kappa \in \mathbb{K}$,

$$
T_{\kappa}\left(\varphi_{\kappa, i}\right)=h_{i}, \quad \forall i \in \mathbb{0} .
$$

Here $\left\{h_{i}\right\}$ is an orthonormal basis for $\mathscr{H}$. Note that we may choose for each $\kappa \in \mathbb{K}$ the same orthonormal basis $\left\{h_{i}\right\}$, because the definition of Riesz bases does not depend on 
the choice of this orthonormal basis. Define $\mathscr{U}$ on $\mathscr{W}$ by

$$
\mathscr{U} f=\sum_{\kappa \in \mathbb{K}} T_{\kappa}[\hat{f}(\kappa)] e_{\kappa} .
$$

Then $\mathscr{U}$ is a bounded linear operator on $\mathscr{W}$. For, applying Parseval's identity to $\mathscr{U} f \in \mathscr{W}$,

$$
\begin{aligned}
\|\mathscr{U} f\|_{\mathscr{H}}^{2} & =\sum_{\kappa \in \mathbb{K}}\|(\mathscr{U} \hat{f})(\kappa)\|_{\mathscr{H}}^{2} \\
& =\sum_{\kappa \in \mathbb{K}}\left\|T_{\kappa}[\hat{f}(\kappa)]\right\|_{\mathscr{H}}^{2} \leqslant \sum_{\kappa \in \mathbb{K}}\left\|T_{\kappa}\right\|^{2}\|\hat{f}(\kappa)\|_{\mathscr{H}}^{2} \leqslant\left(\sup _{\kappa}\left\|T_{\kappa}\right\|^{2}\right)\left(\|f\|_{\mathscr{W}}^{2}\right) .
\end{aligned}
$$

Hence $\mathscr{U}$ is bounded, since $\left\{T_{\kappa}\right\}_{\kappa \in \mathbb{K}}$ is uniformly bounded. The inverse of $\mathscr{U}$ is

$$
\mathscr{U}^{-1} f=\sum_{\kappa \in \mathbb{K}} T_{\kappa}^{-1}[\hat{f}(\kappa)] e_{\kappa} .
$$

For, $(\mathscr{U} \hat{f})(\kappa)=T_{\kappa}[\hat{f}(\kappa)]$ and

$$
\begin{aligned}
\mathscr{U}^{-1}(\mathscr{U} f) & =\sum_{\kappa \in \mathbb{K}} T_{\kappa}^{-1}[(\mathscr{U} \hat{f})(\kappa)] e_{\kappa} \\
& =\sum_{\kappa \in \mathbb{K}} T_{\kappa}^{-1}\left[T_{\kappa} \hat{f}(\kappa)\right] e_{\kappa}=\sum_{\kappa \in \mathbb{K}} \hat{f}(\kappa) e_{\kappa}=f .
\end{aligned}
$$

In a similar manner we check that $\mathscr{U}\left(\mathscr{U}^{-1}(f)\right)=f$. The inverse, $\mathscr{U}^{-1}$, is also bounded, because the family $\left\{T_{\kappa}^{-1}\right\}_{\kappa \in K}$ is uniformly bounded. Finally, since

$$
\left(\mathscr{U}\left[e_{\kappa} \varphi_{\kappa, i}\right]\right) \hat{)}(\kappa)=T_{\kappa} \varphi_{\kappa, i}=h_{i},
$$

it follows that

$$
\mathscr{U}\left(e_{\kappa} \varphi_{\kappa, i}\right)=e_{\kappa} h_{i}, \quad \forall \kappa \in \mathbb{K}, i \in \mathbb{\square} .
$$

Hence $\left\{e_{\kappa} \varphi_{\kappa, i}\right\}$ is a Riesz basis for $\mathscr{W}$.

Note that for a finite index set $\mathbb{K}$ the conditions of this proposition are trivially satisfied. The condition on the uniform boundedness cannot be missed, since a counterexample can be found if $\left\{T_{\kappa}\right\}$ or $\left\{T_{\kappa}^{-1}\right\}$ are not uniformly bounded (e.g., take $T_{\kappa}=(1 / \kappa) \operatorname{Id}_{\mathscr{H}}$, where $\operatorname{Id}_{\mathscr{H}}$ is the identity on $\left.\mathscr{H}\right)$.

We now give an example which shows that there indeed exist Riesz bases that satisfy the conditions of Proposition 3.3.

Example 3.1. Let $\mathscr{H}$ be the Hilbert space $\mathbb{P}_{r}$, let $\mathbb{K}=\mathbb{Z}^{2}$, and $\mathbb{Q}=\mathbb{Z}$. Suppose we have a sequence of real numbers $t_{\kappa, i}$ such that for arbitrary $\kappa \in \mathbb{K}$

$$
\forall i \in \mathbb{}, \quad\left|t_{\kappa, i}-i\right| \leqslant \alpha<\frac{1}{4} .
$$

Then we know by example 2.2 that for fixed $\kappa \in \mathbb{K}$,

$$
\left\{\sqrt{ }(r / \pi) \operatorname{sinc}_{r}\left(t-t_{\kappa, i} \pi / r\right)\right\}_{i \in 0}
$$

is a Riesz basis, that is there exists a linear bijection $T_{\kappa}$ such that

$$
T_{\kappa}\left(\sqrt{ }(r / \pi) \operatorname{sinc}_{r}\left(\cdot-t_{k, i} \pi / r\right)\right)=\sqrt{ }(r / \pi) \operatorname{sinc}_{r}(\cdot-i \pi / r), \quad \forall i \in \mathbb{Q} .
$$


Furthermore, for $\kappa \in \mathbb{K}$ fixed

$$
\left\|T_{\kappa}\right\| \leqslant 1 /(1-\lambda), \quad\left\|T_{\kappa}^{-1}\right\| \leqslant 1+\lambda,
$$

with $\lambda=1-\cos \pi \alpha+\sin \pi \alpha<1$. Hence the families of operators $\left\{T_{\kappa}\right\}_{\kappa \in k}$ and $\left\{T_{\kappa}^{-1}\right\}_{\kappa \in \mathbb{K}}$ are uniformly bounded. By Proposition 3.3

$$
\left\{e_{\kappa}(x)\left[\sqrt{ }(r / \pi) \operatorname{sinc}_{r}\left(t-t_{k, i} \pi / r\right)\right]\right\}_{\kappa \in \mathbb{K}, i \in \Theta}
$$

is a Riesz basis in $L^{2}\left(D, \mathbb{P}_{r}\right)$.

The biorthogonal system of $\left\{e_{\kappa} \varphi_{\kappa, i}\right\}$ has the following form. Suppose $\left\{\varphi_{\kappa, i}\right\}$ satisfies the conditions of Proposition 3.4. Suppose that $\left\{\psi_{\kappa, i}\right\}_{i \in \mathbb{1}}$ is the biorthogonal system of $\left\{\varphi_{\kappa, i}\right\}_{i \in \mathbb{l}}$ for each $\kappa \in \mathbb{K}$. By Proposition 3.4 we have that

$$
\left\{e_{\kappa} \varphi_{\kappa, i}\right\}_{\kappa \in \mathbb{K}, i \in \mathbb{I}}
$$

is a Riesz basis for $\mathscr{W}$. Its (unique) biorthogonal system is

$$
\left\{e_{\kappa} \psi_{\kappa, i}\right\}_{\kappa \in \mathbb{K}, i \in l} \text {, }
$$

which is also a Riesz basis for $\mathscr{W}$.

\subsection{The moment problem in $L^{2}(D, \mathscr{H})$}

Consider the following moment problem in $L^{2}(D, \mathscr{H})$. Suppose we are given a sequence of complex numbers $\left\{g_{\kappa, i}\right\} \in l^{2}(\mathbb{K} \times \mathbb{0})$. Let $\left\{\varphi_{\kappa, i}\right)_{i \in l}$ be a Riesz basis in $\mathscr{H}$, for each $\kappa \in \mathbb{K}$, which satisfies the conditions of Proposition 3.4. We want to find a function $f \in \mathscr{W}$ such that

$$
\left\langle f, e_{\kappa} \varphi_{\kappa, i}\right\rangle_{\mathscr{W}}=g_{\kappa, i}, \quad \forall \kappa \in \mathbb{K}, \forall i \in \mathbb{Q} .
$$

We first prove the following useful equality, for arbitrary $h \in \mathscr{H}$,

and so,

$$
\begin{aligned}
\left\langle f, e_{\kappa} h\right\rangle_{\mathscr{W}} & :=\int_{D}\left\langle f(x), e_{\kappa}(x) h\right\rangle_{\mathscr{H}} \mathrm{d} \mu(x) \\
& =\int_{D}\langle f(x), h\rangle_{\mathscr{H}} \overline{e_{\kappa}(x)} \mathrm{d} \mu(x)=\int_{D}\left\langle f(x) \overline{e_{\kappa}(x)}, h\right\rangle_{\mathscr{H}} \mathrm{d} \mu(x) \\
& =\left\langle\int_{D} f(x) \overline{e_{\kappa}(x)} \mathrm{d} \mu(x), h\right\rangle_{\mathscr{H}}=\langle\hat{f}(\kappa), h\rangle_{\mathscr{H}} .
\end{aligned}
$$

$$
\left\langle f, e_{\kappa} h\right\rangle_{\mathscr{W}}=\langle\hat{f}(\kappa), h\rangle_{\mathscr{H}}, \quad \forall h \in \mathscr{H} .
$$

By (3.6) problem (3.5) can be solved by finding an element $f$ of $\mathscr{W}$ such that

$$
\left\langle\hat{f}(\kappa), \varphi_{\kappa, i}\right\rangle_{\mathscr{H}}=g_{\kappa, i}, \quad \forall \kappa \in \mathbb{K}, i \in \mathbb{Q} .
$$

The method suggested by (3.7) to solve (3.5) is as follows. For each $\kappa \in \mathbb{K}$ find a solution $c_{\kappa} \in \mathscr{H}$ to the problem

$$
\left\langle c_{\kappa}, \varphi_{\kappa, i}\right\rangle_{\mathscr{H}}=g_{\kappa, i}, \quad \forall i \in \mathbb{\mathbb { V }} .
$$

$c_{\kappa}$ is of the form

$$
c_{\kappa}=\sum_{i \in \mathbb{I}} g_{\kappa, i} \psi_{\kappa, i} .
$$


Here for fixed $\kappa \in \mathbb{K}\left\{\psi_{\kappa, i}\right\}_{i \in 1}$ is the biorthogonal system of $\left\{\varphi_{\kappa, i}\right\}_{i \in \mathbb{l}}$, given by

$$
\psi_{\kappa, i}:=\sum_{j \in l}{\overline{\left(G(\kappa)^{-1}\right.}}_{i j} \varphi_{\kappa, j}
$$

The Gram matrix $G(\kappa)$ is (for $\kappa \in \mathbb{K}$ )

$$
(G(\kappa))_{i j}:=\left\langle\varphi_{\kappa, j}, \varphi_{k, i}\right\rangle_{\mathscr{H}}, \quad \forall i, j \in \mathbb{Q} .
$$

If we let the $c_{\kappa}$ play the role of the Fourier coefficients of the solution $f$ to (3.5), $f$ is given by Fourier inversion,

$$
f=\sum_{\kappa \in \mathbb{K}} c_{\kappa} e_{\kappa} .
$$

Problem (3.5) can also be solved directly. Let $\left\{\varphi_{\kappa, i}\right\}$ satisfy the conditions of Proposition 3.3, then $\left\{e_{\kappa} \varphi_{\kappa, i}\right\}$, and its biorthogonal sequence $\left\{e_{\kappa} \psi_{\kappa, i}\right\}$ are Riesz bases. Hence any element of $\mathscr{W}$ can then be written as

$$
f=\sum_{\kappa, i}\left\langle f, e_{\kappa} \varphi_{\kappa, i}\right\rangle_{\mathscr{W}} e_{\kappa} \psi_{\kappa, i} .
$$

The unique solution is then

$$
f=\sum_{\kappa \in \mathbb{K}, i \in \mathbb{1}} g_{\kappa, i} e_{\kappa} \psi_{\kappa, i}
$$

We thus have proven the following theorem.

Theorem 3.4. Let $\left\{\varphi_{\kappa, i}\right\}_{i \in \mathbb{l}}$ (for all $\kappa \in \mathbb{K}$ ) be a Riesz basis for $\mathscr{H}$ such that it satisfies the conditions of Proposition 3.4. Then

$$
f=\sum_{\kappa \in K} c_{\kappa} e_{\kappa}
$$

is a unique solution to (3.5), which lies in $\mathscr{W}$. Here the sequence $c_{\kappa}$ in $\mathscr{H}$ is given by (3.9).

The following proposition is included to characterize the solution in practical situations where the index sets $\mathbb{K}$ and $\mathbb{Q}$ are finite.

Proposition 3.5. Let $\mathbb{K}$ and $\square$ be finite index sets. Suppose that for all $\kappa \in \mathbb{K}$, the sequence $\left\{\varphi_{\kappa, i}\right\}_{i \in \mathbb{I}}$ is linearly independent in $\mathscr{H}$. Then the unique solution of minimum norm to the problem (3.5) is given by (3.12).

Proof. Let $\left\{e_{\kappa}\right\}$ and $\left\{\varphi_{\kappa, i}\right\}$ satisfy the above conditions. If, for all $\kappa \in \mathbb{K},\left\{\varphi_{\kappa, i}\right\}_{i \in \mathbb{K}}$ is linearly independent in $\mathscr{H}$, then $\left\{e_{\kappa} \varphi_{\kappa, i}\right\}_{\kappa \in \mathbb{K}, i \in 1}$ is a linearly independent system in $\mathscr{W}$. Hence, it is a Riesz basis for its linear span. Then it follows by subsection 2.1 that the minimum-norm solution to the moment problem (3.5) is given by 3.12 .

\subsection{The moment problem in $L^{2}\left(D, \mathbb{P}_{r}\right)$}

In the preceding paragraphs we introduced the space $L^{2}(D, \mathscr{H})$, where $\mathscr{H}$ is a separable Hilbert space. In this section we restrict ourselves to the case that $H=\mathbb{P}_{r}$, in order to give a solution to the mixed Fourier-interpolation problem (1.5).

In the following we shall denote a function $f \in L^{2}\left(D, \mathbb{P}_{r}\right)$, as $f(x, t)$. Note that for $x \in D$ fixed $t \rightarrow f(x, t)$ is an element of $\mathbb{P}_{r}$. Its Fourier transform is denoted by $\hat{f}(\kappa, t)$. 
With this notation, by (2.3), and the definition of the $\mathbb{P}_{r}$-valued integral, (3.2) can be rewritten as

$$
\hat{f}(\kappa, t)=\frac{1}{2 \pi} \int_{D} f(x, t) \mathrm{e}^{-\mathrm{i} \kappa x} \mathrm{~d} \mu(x) .
$$

We now show that $(1.5)$ is a special type of moment problem in $L^{2}\left(D, \mathbb{P}_{r}\right)$. Let

$$
\varphi_{\kappa, i}:=\sqrt{ }(r / \pi) \operatorname{sinc}_{r}\left[t-t_{i}(\kappa) \pi / r\right] .
$$

With this notation we have for any $f \in L^{2}\left(D, \mathbb{P}_{r}\right)$, using (3.13), (3.6) and (2.3)

$$
\left\langle f, e_{\kappa} \varphi_{\kappa, i}\right\rangle_{\mathscr{W}}=[\sqrt{ }(\pi / r)] \hat{f}\left(\kappa, t_{i}(\kappa) \pi / r\right) .
$$

Hence, the problem (1.5) can be reformulated as the moment problem (3.5), up to a change of scale.

In the following we give examples of solutions to the moment problem (1.5), as an application of Theorem 3.5 and Proposition 3.6. Example 3.7 deals with the case of countable index sets $\mathbb{0}=\mathbb{Z}$ and $\mathbb{K}=\mathbb{Z}^{2}$.

Example 3.2. Let $\mathbb{Q}=\mathbb{Z}$ and $\mathbb{K}=\mathbb{Z}^{2}$. Suppose that for all $\kappa \in \mathbb{K}$, we have a sequence of real numbers $t_{i}(k)$ such that

$$
\left|i-t_{i}(\kappa)\right| \leqslant \alpha<\frac{1}{4}, \quad \forall i \in \mathbb{0} .
$$

By example 3.1 it follows that, for fixed $\kappa \in \mathbb{K}$,

$$
\varphi_{\kappa, i}:=\operatorname{sinc}_{\pi}\left[t-t_{i}(\kappa)\right]
$$

for $i \in \mathbb{Q}$, is a Riesz basis in $\mathbb{P}_{\pi}$. We know that the unique solution to problem (3.5) is given by (3.12),

$$
\psi_{\kappa, i}=\sum_{j \in l}{\overline{\left(G(\kappa)^{-1}\right.}}_{i j} \varphi_{\kappa, j}
$$

and for $\kappa \in \mathbb{K}$ fixed, the Gram matrix $G(\kappa)$ is

$$
(G(\kappa))_{i j}=\operatorname{sinc}_{\pi}\left[t_{i}(\kappa)-t_{j}(\kappa)\right], \quad \forall i, j \in \mathbb{Q} .
$$

The main issue in the computation of the biorthogonal system $\left\{\psi_{\kappa, i}\right\}$ is the inversion of the Gram matrices (for $\kappa \in \mathbb{K}$ ) $G(\kappa)$. This inversion in the infinitedimensional case will be considered in a future publication. We note for practical purposes that the numerical inversion of the Gram matrix in example 3.2 is the time consuming part in the computation of the solution of the reconstruetion problem.

In the previous example the type $r$ of the space $\mathbb{P}_{r}$ is chosen equal to $\pi$. In the case that both index sets are finite, say $\mathbb{K}=\{0, \ldots, 255\}^{2}$ and $\mathbb{\nabla}=\{1, \ldots, I\}$, we choose $r$ in relation to the spacing of the time points. We assume only that the time points $\left\{t_{i}(\kappa)\right\}_{i \in \mathbb{I}}$ are distinct (for $\left.\kappa \in \mathbb{K}\right)$.

In formula (3.14) the time points at which the data $\left\{g_{\kappa, i}\right\}_{\kappa \in \mathbb{K}, i \in I}$ are measured, are rescaled by a factor $\pi / r$. In the following example we do not know the type $r$ in advance, but we want to determine $r$ according to the spacing of the time points. Assuming the data $\left\{g_{\kappa, i}\right\}$ are measured at the time points $\left\{s_{i}(\kappa)\right\}$, we choose $r$ as follows. 
Example 3.3. For each $\kappa \in \mathbb{K}$ we first choose

$$
r_{\kappa}:=\inf \left\{\frac{\pi}{\left|s_{i}(\kappa)-s_{i+1}(\kappa)\right|} \mid i \in \mathbb{Q}\right\} .
$$

Note that if the consecutive sampling points were lying a distance

$$
h_{\kappa}:=\sup \left\{\left|s_{i}(\kappa)-s_{i+1}(\kappa)\right| \mid i=1,2, \ldots, I-1\right\}
$$

apart, then $h_{\kappa}$ would be the Nyquist rate that corresponds to the type $r_{\kappa}$ (cf. Natterer [13], p. 56). If we define $r:=\sup \left\{r_{\kappa} \mid(\kappa) \in \mathbb{K}\right\}$, then $\mathbb{P}_{r_{\kappa}} \subset \mathbb{P}_{r}$. Define $t_{i}(\kappa)=s_{i}(\kappa) r_{\kappa} / \pi$. Since for each $\kappa \in \mathbb{K}$ the finite sequence of vectors

$$
\varphi_{\kappa, i}:=\left(r_{\kappa} / \pi\right) \operatorname{sinc}_{r_{\kappa}}\left[t-t_{i}(\kappa) \pi / r_{\kappa}\right],
$$

for $i \in \mathbb{U}$, lies in $\mathbb{P}_{r_{\kappa}}$, it also lies in the larger space $\mathbb{P}_{r}$. A solution to (3.5) in $L^{2}\left(D, \mathbb{P}_{r}\right)$ may be given as follows. Let $\left\{t_{i}(\kappa)\right\}_{i \in l}$, for all $\kappa \in \mathbb{K}$, be a sequence of distinct, arbitrarily chosen, real numbers. Let $\mathbb{Q}=\{1,2, \ldots, I\}$ and put

$$
\varphi_{\kappa, i}:=\left(r_{\kappa} / \pi\right) \operatorname{sinc}_{r_{\kappa}}\left[t-t_{i}(\kappa) \pi / r_{\kappa}\right],
$$

which is, for $\kappa \in \mathbb{K}$ fixed, a linearly independent system in $\mathbb{P}_{r}$. Define

$$
\Phi_{\kappa, i}:=e_{\kappa} \varphi_{\kappa, i},
$$

which is a linearly independent system in $L^{2}\left(D, \mathbb{P}_{r}\right)$. By Proposition 3.6 the minimumnorm solution $f \in \operatorname{span}\left\{\Phi_{\kappa, i}\right\}$, which satisfies (3.5),

$$
\left\langle f, \Phi_{\kappa, i}\right\rangle_{\mathbf{P}_{r}}=g_{\kappa, i}, \quad \forall \kappa \in \mathbb{K}, \forall i \in \mathbb{U},
$$

is given by formula (3.12). The rest is the same as in example 3.2. The biorthogonal vectors $\psi_{\kappa, i}$ are given by (3.10) and the Gram matrix is defined by (3.15).

\section{Conclusions and additional remarks}

In this paper we have solved the mixed Fourier-interpolation problem by means of Riesz bases. The idea was to perform for each frequency $\kappa$ a sinc interpolation along the time axis. The (minimum-norm) solution to (1.5) is obtained by means of a Fourier inversion. The reason why we do not have to perform interpolation in the frequency plane is the orthogonality of the exponentials $\left\{\mathrm{e}^{\mathrm{i} \kappa x}\right\}_{\kappa \in \mathbb{K}}$. If we had a priori information about the measured object, expressed in terms of a particular norm, then the exponentials need not be an orthonormal system and the situation will be different. In such a case we have to perform interpolation in the frequency plane in order to obtain the minimum-norm solution.

Instead of using sinc interpolation, one could use spline interpolation, which will give good results in the practice of MRI. The application of the examples (and algorithms) to test images, as well as to real MRI data, will be considered in a future publication.

\section{Acknowledgements}

I gratefully thank the Philips Medical Systems Division for explaining many of the practical aspects of MRI and retrospective synchronization. I would like to thank Dr J. B. T. M. Roerdink and Dr. H. J. A. M. 
Heijmans for a large number of comments and for their thorough reading of the manuscript. I am indebted to Professor Dr G. Y. Nieuwland for stimulating discussions about this subject.

\section{References}

1. Akhiezer, N. I., The Classical Moment Problem Oliver \& Boyd, Edinburgh, 1965.

2. Balakrishnan, A. V., Applied Functional Analysis, Springer, Berlin, 1976.

3. Bertero, M.,' Regularization methods for linear inverse problems' Inverse Problems Lecture Notes in Mathematics No. 1225, Eds A. Dold and B. Eckman, Springer, Berlin, 1986, pp 52-113.

4. Bohning, D. E., Cardiac gating strategies, New Concepts in Cardiac Imaging, Ch. 11, Yearbook Medical Publishers, 1988.

5. Butzer, P. L., Splettstösser, W. and Stens, R. L., 'The sampling theorem and linear prediction in signal analysis', Jahresbericht der Deutsche Mathematik Verein, 90, 1-70 (1988).

6. Foias, C. and Nagy, B. Sz., Harmonic Analysis of Operators on Hilbert Spaces, North-Holland, Amsterdam, 1970.

7. Gohberg, I. C. and Goldberg, S., Basic Operator Theory, Birkhäuser, Boston, 1980.

8. Gohberg, I. C. and Krein, M. G., Introduction to the Theory of Linear Nonselfadjoint Operators, Translations of Mathematical Monographs, Vol. 18, AMS, Providence, RI, 1969.

9. Hille, E. and Phillips, R. S., Functional Analysis and Semigroups, Colloquium Publications, Vol. XXXI, AMS, Providence, RI, 1957.

10. Hinshaw, W. S. and Lent, A. H., 'An introduction to NMR imaging: from the Bloch equation to the imaging equation', Proc. IEEE, 71, (3), (1983).

11. Landau, H. J. (ed.), Moments in Mathematics, Proceedings of Symposia in Applied Mathematics, Vol. 37, AMS, Providence, RI, 1987.

12. Mansfield, P. and Morris, P. G., NMR Imaging in Biomedicine, Academic, New York, 1982.

13. Natterer, F., The Mathematics of Computerized Tomography Wiley, Chichester, 1986.

14. Rudin, W., Functional Analysis, McGraw-Hill, New Delhi, 1974.

15. Shapiro, H. S., Topics in Approximation Theory, Lecture Notes in Mathematics No. 187, Eds A. Dold and B. Eckman, Springer, Berlin, 1971.

16. Young, R. M., An Introduction to Nonharmonic Fourier Series, Academic, New York, 1980.

17. Zwaan, M., 'Dynamic MRI reconstruction as a moment problem. Part I. The beating heart: a problem formulation', Report AM-R8905, March-1989.

18. Zwaan, M., 'Dynamic MRI reconstruction as a moment problem. Part II. Riesz bases in $L^{2}$-spaces of vector valued functions', Report AM-R8907, April 1989. 